\title{
Análise de um Jogo Educacional para Mensuração de Habilidades Cognitivas Preditoras do Desenvolvimento de Leitura em Crianças
}

\author{
Jucelio Soares dos Santos ${ }^{1}$, Monilly Ramos Araújo de Melo ${ }^{1}$ Dalton Dario Serey \\ Guerrero $^{1}$, Jorge César Abrantes Figueiredo ${ }^{1}$, Carla Alexandra Silva Moita \\ Minervino $^{2}$ \\ ${ }^{1}$ Universidade Federal de Campina Grande \\ 2 Universidade Federal da Paraíba \\ jucelio@copin.ufcg.edu.br, monillyramos@gmail.com, \{dalton, \\ abrantes\}@dsc.ufcg.edu.br, carlamoitaminervino@gmail.com
}

\begin{abstract}
One of the main challenges for schools is to identify the limitations of reading in children. This reality could be improved if educational professionals had a resource that would help them detect limitations in reading, guiding appropriate intervention methods for the child. To do so, we developed an educational game for mobile devices, designed appropriately to measure predictive reading abilities. In this article, we present a study to verify if the proposed game can distinguish the performance of two sample groups (clinical and school) in relation to children's ability and response time in tasks of phonological awareness (alliteration, segmentation and rhyme), memory visual and visual-motor coordination. As results, in all the tasks of the game, the children belonging to the school group performed better and significantly when compared to the children of the same age group and gender of the clinical group, and this observation gives us strong indications that the game can distinguish children with strong and weak reading ability.
\end{abstract}

Resumo. Um dos principais desafios das escolas é identificar as limitações de leitura em crianças. Esta realidade poderia ser melhorada se os profissionais da educação dispusessem de um recurso que os auxiliassem a detectar limitações na leitura, orientando em métodos interventivos adequados a criança. Para tanto, desenvolvemos um jogo educacional para dispositivos móveis, projetado adequadamente para mensurar as habilidades preditoras de leitura. Neste artigo, apresentamos um estudo para verificar se o jogo proposto consegue distinguir o desempenho de dois grupos amostrais (clínico e escolar) em relação a habilidade e o tempo de resposta das crianças em tarefas de consciência fonológica (aliteração, segmentação e rima), memória visual e coordenação viso-motora. Como resultados, em todas as tarefas do jogo, as crianças pertencentes ao grupo escolar obtiveram desempenho melhor e significativo quando comparadas às crianças de mesma faixa etária e gênero do grupo clínico, e tal observação nos dá fortes indícios de que o jogo consegue distinguir crianças com forte e fraca habilidade de leitura. 
VII Congresso Brasileiro de Informática na Educação (CBIE 2018)

Anais do XXIV Workshop de Informática na Escola (WIE 2018)

\section{Introdução}

O domínio de atuação da Ciência da Computação é imensamente vasto, incluindo desde os seus fundamentos teóricos e algorítmicos até os mais avançados desenvolvimentos em robótica e automação inteligente. Porém, existem alguns problemas difíceis de serem resolvidos, pois requerem conhecimentos acumulados para a detecção de particularidades.

Uma das áreas afetadas por esse problema é a Psicologia, pois exige que um profissional capacitado observe o comportamento e as reações do seu paciente para chegar a um pré-diagnóstico. Nessa área, podemos citar a dislexia ${ }^{1}$, transtorno cujos recursos computacionais, especializados, válidos e comprovados estatisticamente do seu uso são escassos para auxiliar em seu rastreio. O rastreio é a detecção presuntiva do distúrbio não reconhecida de defeito, realizando exames ou outros procedimentos que determinarão a probabilidade do distúrbio. Este processo não se destina a ser diagnóstico e aqueles com resultados positivos ou suspeitos devem ser encaminhados para um psicólogo para o diagnóstico e o tratamento necessário [Ekhsan et al. 2012].

Rastrear precocemente escolares em situações de risco, durante a fase de alfabetização, torna-se uma atividade importante para que haja uma melhor estimulação no cérebro da criança. Dessa forma, a busca por programas para identificar as limitações na leitura torna-se um desafio aos profissionais da educação. Para esses casos é importante considerar o uso de programas de triagem estruturados e de eficácia comprovada para identificar crianças com fraca/forte habilidade na leitura.

Devido a esta carência, desenvolvemos um jogo educacional intitulado "Os Niridianos no inacreditável Mar de Letras" adaptado do Teste de Habilidades Preditoras de Leitura (THPL) [Moita, 2013]. Esse jogo é administrado por um algoritmo de seleção adaptativa de itens que mensura as seguintes habilidades linguísticas: aliteração, segmentação, memória visual e rima. Esta habilidade é estimada conforme a Teoria de Resposta ao Item (TRI).

Na dislexia, os jogos são fortes aliados para estimular a criação de habilidades na leitura, reforçando a aprendizagem de forma estruturada e multissensorial. Através dos jogos é possível que a criança revise constantemente um assunto, além de proporcionar um trabalho individual seguindo o ritmo do aluno [Santos et al, 2014]. Além disso, é possível que o indivíduo faça o uso de diferentes atividades, estimulando o desenvolvimento das habilidades cognitivas e emocionais [Amaral e Costa, 2011].

Neste artigo apresentamos um estudo para verificar se o jogo desenvolvido nesta pesquisa consegue distinguir o desempenho de crianças com fraca/forte habilidade em consciência fonológica (aliteração, segmentação e rima), memória visual e coordenação viso-motora. O presente estudo pretende contribuir com a investigação de uma solução mais próxima e pertinente a uma das realidades singulares do ensino, identificando crianças com/sem risco de apresentarem dislexia. Os resultados desse estudo poderão ser usados para uma prática baseada em evidências nas escolas brasileiras.

\footnotetext{
${ }^{1}$ Dislexia é um déficit no processamento fonológico responsável pela dificuldade na decodificação e relação entre letras e sons [Catts and Kahmi 1999].
} 
VII Congresso Brasileiro de Informática na Educação (CBIE 2018)

Anais do XXIV Workshop de Informática na Escola (WIE 2018)

\section{Metodologia}

O método de pesquisa deste trabalho é um experimento com intuito de verificar se o jogo proposto neste trabalho consegue distinguir crianças com fraca/forte habilidade em tarefas preditoras da leitura. Este trabalho busca responder as seguintes questões pesquisas: O jogo “Os Niridianos no Inacreditável Mar de Letras” consegue distinguir crianças com fraca/forte habilidade em suas tarefas? Existe diferença no tempo de resposta nas tarefas do jogo em crianças com fraca/forte habilidade?

\subsection{Ferramenta utilizada}

O instrumento utilizado neste estudo foi o jogo “Os Niridianos no Inacreditável Mar de Letras” desenvolvido a partir das tarefas de aliteração, segmentação, memória visual e rima do THPL [Moita, 2013]. Além destas tarefas, o jogo explora, devido a sua aplicabilidade, a tarefa de coordenação viso-motora.

A atividade de aliteração permite avaliar a habilidade da criança na identificação de fonemas idênticos ou parecidos no início de várias palavras. No jogo é apresentado uma figura-estímulo (com a representação sonora) e três figuras-teste; entre as figurasteste uma aliterava com a figura-estímulo, conforme a Figura 1. A atividade de segmentação permite avaliar a habilidade da criança na identificação do número de sílabas da palavra em causa. No jogo é apresentada uma figura-estímulo (com a representação sonora) e são apresentadas cinco possibilidades de resposta que podem corresponder a quantidade de segmentos, havendo apenas uma possibilidade correta de resposta. A atividade de memória visual, por sua vez, permite avaliar a habilidade de memória visual da criança em um curto intervalo de tempo. No jogo se configura na observação de uma figura em um curto intervalo de tempo e, em seguida, na sua identificação num conjunto maior de desenhos. A atividade de rima permite avaliar a habilidade da criança na identificação da palavra que contém a mesma terminação. No jogo é apresentado uma figura-estímulo (com a representação sonora) e três figurasteste; entre as figuras-teste uma rimava com a figura-estímulo.

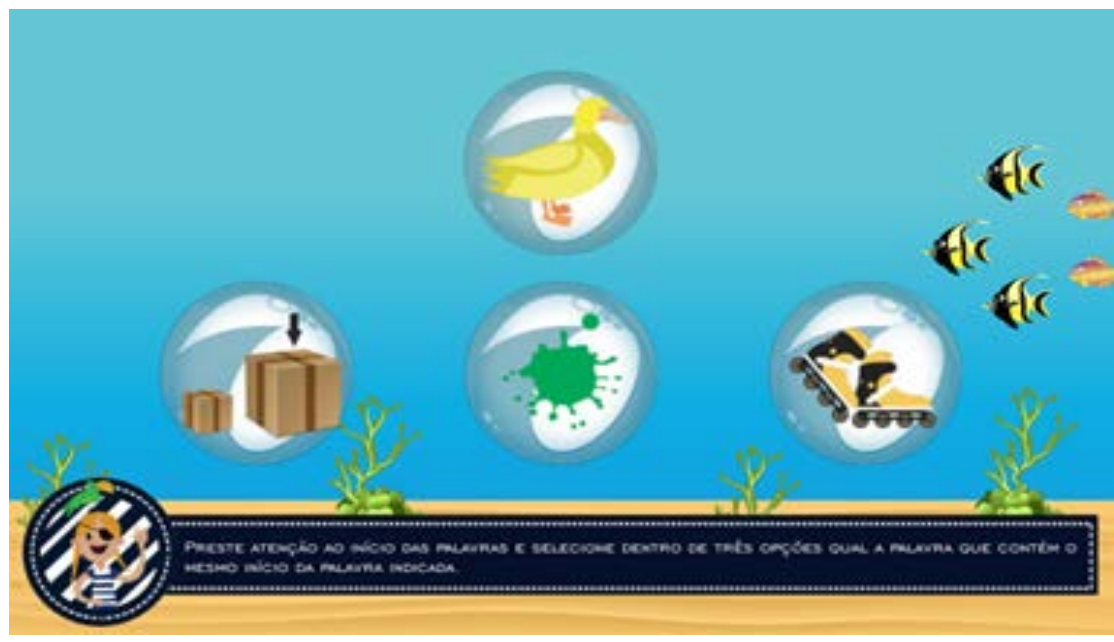

Figura 1. Exemplo da Tarefa Aliteração

A atividade de coordenação viso-motora permite avaliar a habilidade da criança no que tange ao direcionamento intencional dos olhos, controlando a acuidade visual, ou seja, a capacidade de ver e diferenciar objetos apresentados no campo visual com 
VII Congresso Brasileiro de Informática na Educação (CBIE 2018)

Anais do XXIV Workshop de Informática na Escola (WIE 2018)

significado e precisão. É pedido a criança para recuperar o tesouro no menor tempo e colidir com as cavernas o mínimo de vezes, conforme a Figura 2.

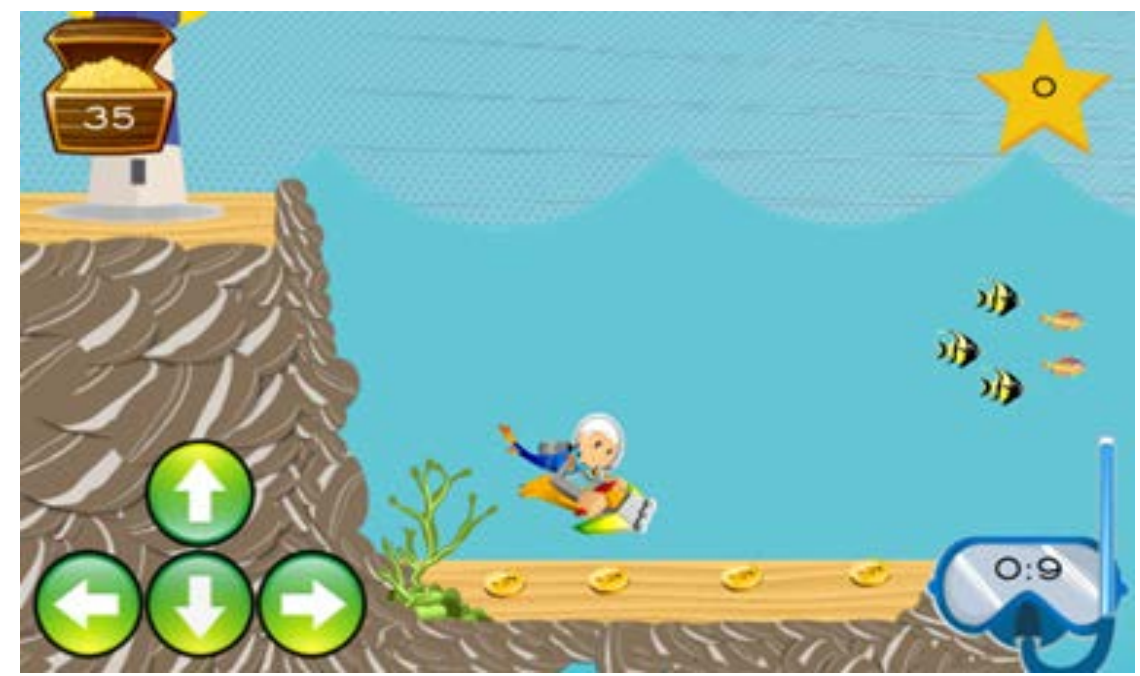

Figura 2. Exemplo da Tarefa Coordenação Viso-Motora

\subsection{Participantes}

Participaram deste estudo, 38 crianças do ensino fundamental I da rede pública de ensino do munícipio de Campina Grande-PB, distribuídos em dois grupos: i) Grupo clínico composto por 19 crianças com diagnóstico de dislexia, sendo 78,94\% gênero masculino e 21,05\% do gênero feminino, com média etária de 10 anos e 4 meses. Esses escolares foram submetidos à avaliação fonoaudiológica na Fundação Papel Marchê, verificando-se sinais de dislexia, ou seja, presença de transtorno fonológico na fala, leitura e escrita, alterações em habilidades fonológicas e silábicas e, presença de histórico familiar positivo para dislexia; ii) Grupo escolar composto por 19 crianças com bom desempenho escolar indicadas pelas professoras mediante o desempenho satisfatório em dois bimestres consecutivos. Estas crianças foram pareadas segundo gênero e média etária com o grupo clínico.

Os critérios de inclusão considerados para o grupo clínico foram a assinatura do Termo de Assentimento e a suspeita diagnóstica de dislexia ou a suspeita de ausência de outro distúrbio de aprendizagem, descritos em prontuário pelas avaliações realizadas na Fundação Papel Marchê. Já os critérios de exclusão foram a não assinatura do Termo de Assentimento pelos pais ou responsáveis dos menores, presença de qualquer déficit sensorial (e.g., visual, auditivo) ou suspeita de transtorno global de desenvolvimento, descrito em prontuário a partir dos resultados das avaliações realizadas na Fundação Papel Marchê.

\subsection{Procedimento de Execução}

A pesquisa decorreu na Fundação Papel Marchê e em 6 (seis) escolas ${ }^{2}$ públicas

\footnotetext{
2 Escola Municipal de Ensino Fundamental Joselita Brasileiro, Escola Municipal de Ensino Fundamental Melo Leitão, Escola Municipal de Ensino Fundamental Semente de Luz, Escola Municipal de Ensino Fundamental Professora Maria Anunciada Bezerra, Escola Municipal de Ensino Fundamental Maria Minervina de Figueiredo e Escola Municipal de Ensino Fundamental Mariinha Borborema.
} 
municipais localizadas na cidade de Campina Grande, Paraíba, Brasil. Tais crianças foram consentidas pelos seus responsáveis que assinaram o termo de assentimento, liberando o menor para participar como voluntário do estudo.

As crianças consentidas foram encaminhadas para uma sala com boa iluminação e a aplicação sucedeu em grupos de 6 (seis) crianças. Ao chegar na sala, a criança foi auxiliada por um aplicador. Inicialmente, foram recolhidas pelos aplicadores algumas informações da criança, tais como: nome, idade, data de nascimento, escolaridade, nome da escola, além de informar se a criança lê, se alguém lê para ela e se ela possui livros em casa. Em seguida, o aplicador entregou o dispositivo móvel (tablets) para a criança iniciar a avaliação das habilidades preditoras da leitura, de acordo com o exemplo de aplicação da Figura 3.

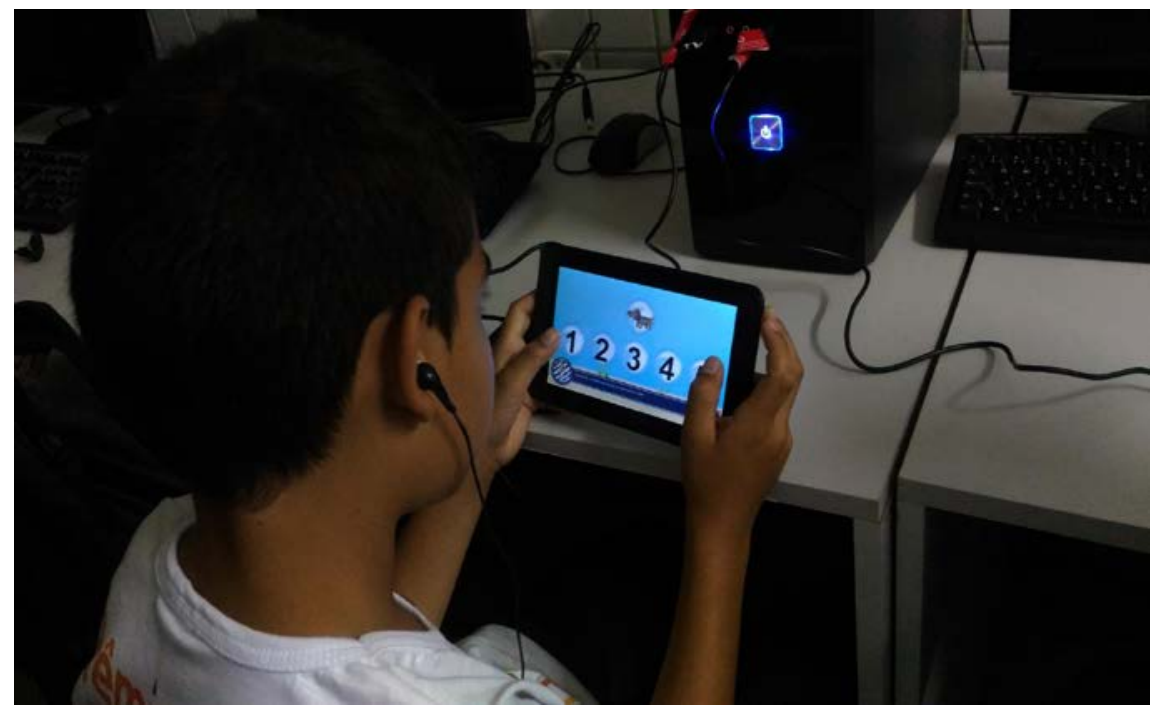

Figura 3. Exemplo da aplicação do jogo “Os Niridianos no Inacreditável Mar de Letras” para tarefa Segmentação

O instrumento é autoaplicável, portanto, a criança escutava as instruções correspondentes ao item e selecionava a resposta que acreditava ser a correta.

\subsection{Análise dos dados}

Durante o procedimento de organização e tratamento dos dados, utilizamos o software Rstudio como ferramenta central no processo de análise de dados, tendo em vista o poder desta ferramenta em realizar cálculos estatísticos complexos, auxiliando no processamento de transformação de dados em informações.

Coletamos os dados dos grupos clínico e escolar a fim de observar se as hipóteses nulas poderiam ser refutadas. Para a análise foram utilizados testes estatísticos não paramétricos devido aos dados não apresentarem uma distribuição normal.

Para analisar as habilidades das tarefas aliteração, segmentação, memória visual e rima; bem como o número de objetos colididos e recolhidos da tarefa coordenação viso-motora; e, o tempo de resposta em todas as tarefas, adotamos o teste não paramétrico wilcoxon para verificar se existe diferença significativa entre as medianas. Adotamos um nível de significância de 0.05 que corresponde a um nível de confiança de $95 \%$. 
VII Congresso Brasileiro de Informática na Educação (CBIE 2018)

Anais do XXIV Workshop de Informática na Escola (WIE 2018)

\section{Resultados e Discussões}

Procuramos dimensionar o desempenho das crianças mediante os parâmetros: a estimativa da habilidade das crianças e o seu tempo de resposta para cada tarefa.

\subsection{Habilidade das crianças}

A Figura 4 representa o boxplot das habilidades estimadas para cada grupo de indivíduos por tarefa. Podemos perceber que nas tarefas aliteração, segmentação, memória visual e rima muitos indivíduos da amostra clínica tem habilidade inferiores quando comparadas a amostra escolar.

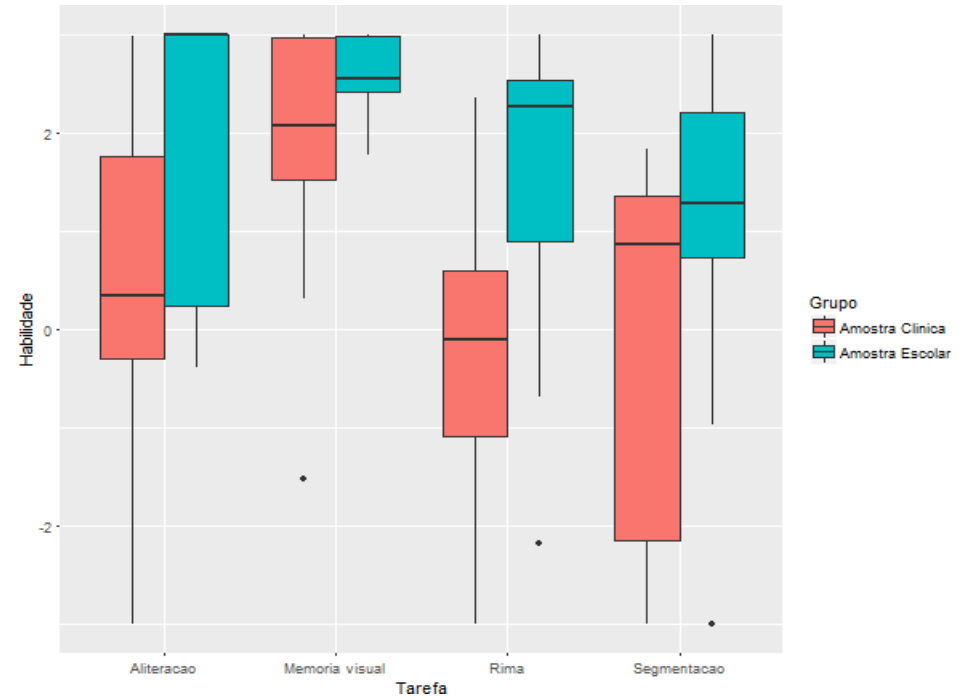

Figura 4. Gráfico boxplot da habilidade das tarefas aliteração, segmentação, memória visual e rima, entre os grupos

Já na atividade de coordenação viso-motora, podemos perceber na Figura 5, que muitos indivíduos do grupo clínico colidiram mais nos objetos do jogo (cavernas, pedras, barcos e magmas) em comparação ao grupo escolar. Já na quantidade de objetos recolhidos (tesouro), alguns indivíduos do grupo clínico recolheram em pouca quantidade.

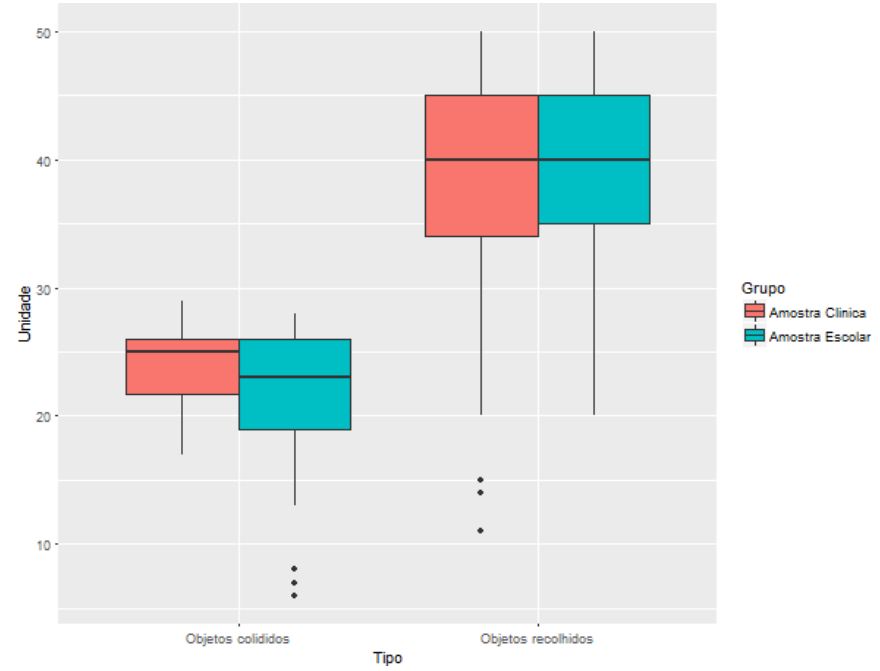

Figura 5. Gráfico boxplot da habilidade da tarefa coordenação viso-motora, entre os grupos 
VII Congresso Brasileiro de Informática na Educação (CBIE 2018)

Anais do XXIV Workshop de Informática na Escola (WIE 2018)

Após recolher os dados, todas as variáveis foram testadas quanto à sua normalidade (com nível de significância de p menor que 0.05), e diante da distribuição não normal dos dados, foi utilizado o teste de wilcoxon a fim de comparar a habilidade estimada das crianças pertencentes aos dois grupos e que responderam às tarefas de aliteração, segmentação, memória visual e rima, conforme a Tabela 1. Também comparamos os grupos em relação a coordenação viso motora, conforme a Tabela 2.

Tabela 1. Análise da habilidade das tarefas aliteração, segmentação, memória visual e rima, entre os grupos

\begin{tabular}{|l|c|c|c|c|c|}
\hline \multirow{2}{*}{ Tarefa } & \multicolumn{2}{|c|}{ Amostra Clínica } & \multicolumn{2}{c|}{ Amostra Escolar } & $\begin{array}{c}\text { Significância } \\
\text { (p) }\end{array}$ \\
\cline { 2 - 5 } & Mediana & Dp & Mediana & Dp & \\
\hline Aliteração & 0,345 & 1,604 & 3 & 1,454 & 0,0222 \\
\hline Segmentação & 0,862 & 1,888 & 1,286 & 1,482 & 0,0370 \\
\hline Memória Visual & 2,081 & 1,176 & 2,550 & 0,366 & 0,0276 \\
\hline Rima & $-0,115$ & 1,546 & 2,270 & 1,473 & 0,0010 \\
\hline
\end{tabular}

Tabela 2. Análise da habilidade da tarefa coordenação viso-motora, entre os grupos

\begin{tabular}{|l|c|c|c|c|c|}
\hline \multirow{2}{*}{ Parâmetros } & \multicolumn{3}{|c|}{ Amostra Clínica } & \multicolumn{3}{|c|}{ Amostra Escolar } & $\begin{array}{c}\text { Significância } \\
\text { (p) }\end{array}$ \\
\cline { 2 - 5 } & Mediana & Dp & Mediana & Dp & \\
\hline Objetos colididos & 25 & 2,972 & 23 & 4,836 & 0,0002 \\
\hline Objetos recolhidos & 40 & 9,810 & 40 & 7,755 & 0,0326 \\
\hline
\end{tabular}

Consideramos como hipótese nula a habilidade estimada das crianças no grupo escolar é a mesma quando comparadas às crianças do grupo clínico nas tarefas de aliteração, segmentação, memória visual, rima e coordenação viso-motora. Queremos verificar se as crianças do grupo escolar têm suas habilidades estimadas maiores que as crianças do grupo clínico nas tarefas aliteração, segmentação, memória visual e rima. Já na tarefa coordenação viso-motora, consideramos dois parâmetros: objetos colididos e objetos recolhidos. Queremos veremos verificar se as crianças do grupo clínico colidiram mais nos objetos e recolheu menos tesouros quando comparadas ao grupo escolar.

Pôde-se inferir, de acordo com as habilidades estimadas pelo jogo, que as crianças do grupo escolar obtiveram desempenho melhor nas tarefas de aliteração, segmentação, memória visual e rima quando comparadas ao grupo clínico e que esta diferença foi estatisticamente significativa. Portanto, temos fortes indícios de que o jogo consegue distinguir crianças com forte e fraca habilidade em atividades preditoras de leitura.

No que diz respeito a coordenação viso-motora, as crianças do grupo escolar obtiveram melhor desempenho em relação ao grupo clínico, de forma significativa, ou seja, colidiu menos nos objetos e colheu mais tesouros. Tais dados nos fornecem fortes indícios que o jogo consegue distinguir características essenciais em relação a coordenação viso-motora. 
VII Congresso Brasileiro de Informática na Educação (CBIE 2018)

Anais do XXIV Workshop de Informática na Escola (WIE 2018)

\subsection{Tempo de resposta das crianças}

Na segunda parte do estudo, comparamos o tempo de resposta das crianças entre os grupos. Como podemos observar na Figura 6, as crianças do grupo clínico levaram mais tempo para concluir as tarefas do jogo.

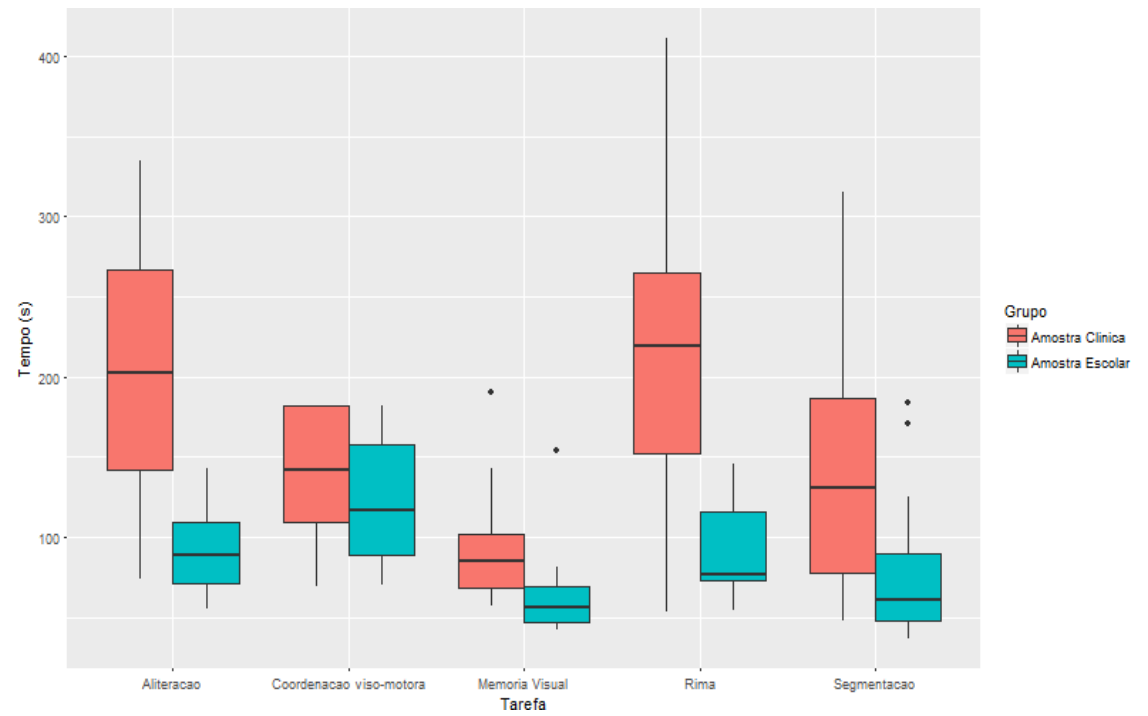

Figura 6. Gráfico boxplot do tempo de resposta das tarefas aliteração, segmentação, memória visual, rima e coordenação viso-motora, entre os grupos

Após serem recolhidos os dados realizamos o teste de normalidade nas variáveis, e diante da distribuição não normal dos dados, foi utilizado o teste de wilcoxon a fim de comparar o tempo de resposta das crianças dos dois grupos que responderam às tarefas de aliteração, segmentação, memória visual e rima, conforme a Tabela 3.

Tabela 3. Análise do tempo de resposta das tarefas aliteração, segmentação, memória visual, rima e coordenação viso-motora, entre os grupos

\begin{tabular}{|c|c|c|c|c|c|}
\hline \multirow[t]{2}{*}{ Tarefa } & \multicolumn{2}{|c|}{ Amostra Clínica } & \multicolumn{2}{|c|}{ Amostra Escolar } & \multirow{2}{*}{$\begin{array}{c}\text { Significância } \\
\text { (p) }\end{array}$} \\
\hline & Mediana & $\mathrm{Dp}$ & Mediana & $\mathrm{Dp}$ & \\
\hline Aliteração & 202 & 79,26 & 89 & 27,21 & 0,0002 \\
\hline Segmentação & 131 & 75,91 & 61 & 42,23 & 0,0016 \\
\hline Memória Visual & 85 & 32,75 & 56 & 25,43 & 0,0016 \\
\hline Rima & 219 & 87,25 & 77 & 28,10 & 0,0001 \\
\hline Coordenação viso-motora & 142 & 37,48 & 116,5 & 39,14 & 0,0052 \\
\hline
\end{tabular}

Consideramos como hipótese nula o tempo de resposta das crianças no grupo escolar é o mesmo quando comparadas às crianças do grupo clínico nas tarefas de aliteração, segmentação, memória visual, rima e coordenação viso-motora. Queremos verificar se as crianças do grupo escolar levaram menos tempo em responder as tarefas quando comparadas às crianças do grupo clínico.

Do exposto acima, concluímos que as crianças do grupo escolar levaram menos tempo, significativamente em todas as tarefas do jogo, quando comparadas com o tempo de resposta das crianças do grupo clínico. 
VII Congresso Brasileiro de Informática na Educação (CBIE 2018)

Anais do XXIV Workshop de Informática na Escola (WIE 2018)

\section{Conclusões}

Neste artigo apresentamos um estudo para comparar o desempenho de dois grupos amostrais (clínico e escolar) nas seguintes dimensões: habilidade e tempo de resposta das crianças. Na dimensão habilidade das crianças, com o recurso da estimativa gerada pelo jogo, concluímos que nas tarefas de aliteração, segmentação, memória visual e rima, a criança do grupo escolar teve desempenho melhor e significativo quando comparada a criança de mesma faixa etária e gênero do grupo clínico. E, tal observações nos dá fortes indícios de que o jogo consegue distinguir crianças com forte e fraca habilidade. Já na coordenação viso-motora, o grupo escolar obteve melhor desempenho e significativo em relação ao grupo clínico, ou seja, colidiu menos nos objetos e colheu mais objetos. Quanto ao tempo, observamos que o grupo escolar levou menos tempo para realizar todas tarefas do jogo, significativamente, quando comparado ao grupo clínico, ou seja, para atingir os objetivos propostos, as crianças pertencentes ao grupo clínico se esforçam mais do que as que pertencem ao grupo escolar.

\section{Referências}

Amaral, N. C. and Costa, P. C. (2011). A informática como auxiliar no tratamento da dislexia. Revista Científica Eletrônica de Pedagogia, (17).

Andrade, D., Tavares, H., and Valle, R. C. (2000). Teoria da Resposta ao Item: Conceitos e Aplicações. ABE, São Paulo, 1 edition.

Catts, H. W. and Kahmi, A. G. (1999). Language and Reading Disabilities. Allyn Bacon, Boston, 1 edition.

Ekhsan, H. M., Ahmad, S. Z., Halim, S. A., Hamid, J. N., and Mansor, N. H. (2012). The implementation of interactive multimedia in early screening of dyslexia. International Conference on Innovation Management and Technology Research, pages

566-569.

Moita, P. M. S. (2013). Avaliação adaptativa em dispositivos móveis das habilidades cognitivas preditoras do desenvolvimento de leitura em crianças. Master's thesis, Universidade de Lisboa. Instituto de Educação. Faculdade de Ciências. Programa de Pós-Graduação em Tecnologias e Metodologias em E-learning, Lisboa.

Santos, J. S.; Costa, R. A.; Souza, R. P.; Pereira, I. B. and Pereira, R. S. O. (2014). Proposta de um jogo educacional para alfabetização de crianças com dislexia. Workshop de Informática na Escola, (20):457-466. 